\title{
Soliton-induced spectrally uniform ion line power enhancements at the ionospheric $F$ region peak
}

\author{
Jonas Ekeberg $^{1}$, Gudmund Wannberg ${ }^{1}$, Lars Eliasson ${ }^{1}$, and Ingemar Häggström² \\ ${ }^{1}$ Swedish Institute of Space Physics, P.O. Box 812, SE-981 28 Kiruna, Sweden \\ ${ }^{2}$ EISCAT Scientific Association, P.O. Box 812, SE-981 28 Kiruna, Sweden
}

(Received April 18, 2011; Revised February 24, 2012; Accepted February 28, 2012; Online published August 16, 2012)

\begin{abstract}
We present European Incoherent Scatter (EISCAT) observations of spectrally uniform ion line power enhancements (SUIPE), where the up- and downshifted shoulder and the spectral valley between them are enhanced simultaneously and equally. We have identified 48 cases of this type of ion line enhancement in data from the EISCAT Svalbard radar taken during the International Polar Year (extending from March 2007 to the end of February 2008). The SUIPEs are observed at altitudes between $210 \mathrm{~km}$ and $280 \mathrm{~km}$ with a standard deviation of $9 \%$ of the average occurrence height $230 \mathrm{~km}$. The power enhancements are one order of magnitude above the thermal level. The SUIPEs occur at the ionospheric $F$ region density peak with $85 \%$ of the cases located within $10 \mathrm{~km}$ of the peak. These characteristics are in good agreement with the predictions of a recently published model for soliton-induced ion-line enhancements at the $F$ region peak. The SUIPE occurrence shows a clear preference for magnetically disturbed conditions, with the likelihood of occurrence increasing with increasing $K$ index. A majority of the events occur in the magnetic evening to pre-midnight sector.
\end{abstract}

Key words: Auroral ionosphere, radar observations, plasma waves and instabilities, solitons and solitary waves.

\section{Introduction}

Incoherent scatter radar spectra from the ionosphere are formed predominantly by scattering of radio waves off ionacoustic and Langmuir waves. This results in the ion- and plasma lines. Due to Landau damping, the ion lines are broadened and merge into a "double-humped" line with a downshifted and an upshifted shoulder, corresponding to scattering off ion-acoustic waves propagating respectively away from and towards the radar.

Occasionally, when the radar beam is close to parallel to the geomagnetic field, one or both of these shoulders are enhanced up to 4-5 orders of magnitude above the thermal level (Grydeland et al., 2004); this is referred to as naturally enhanced ion-acoustic lines (NEIAL). NEIAL are interpreted as scattering off destabilised ion-acoustic waves and the process was first described by Rosenbluth and Rostoker (1962).

NEIAL were first observed by the Millstone Hill incoherent scatter radar (Foster et al., 1988) and were later seen with the European Incoherent Scatter (EISCAT) UHF (Rietveld et al., 1991), the EISCAT VHF (Collis et al., 1991) and the EISCAT Svalbard Radar (ESR) (Buchert et al., 1999). For further details on NEIAL observations and suggested generation mechanisms, please refer to the review by Sedgemore-Schulthess and St.-Maurice (2001) and references therein.

In the statistical study by Rietveld et al. (1996), NEIAL

Copyright (c) The Society of Geomagnetism and Earth, Planetary and Space Sciences (SGEPSS); The Seismological Society of Japan; The Volcanological Society of Japan; The Geodetic Society of Japan; The Japanese Society for Planetary Sciences; TERRAPUB.

doi:10.5047/eps.2012.02.005 were typically observed in the $F$ region with an occurrence peak around $500 \mathrm{~km}$ altitude and no cases below $300 \mathrm{~km}$ altitude with both ion-acoustic shoulders enhanced simultaneously were found. NEIAL are often seen simultaneously over a height range of several $100 \mathrm{~km}$ (e.g., Rietveld et al., 1991) and have been observed up to $1900 \mathrm{~km}$ altitude (Ogawa et al., 2006).

Michell et al. (2008) reported observations of enhanced ion lines at low altitudes $(250-350 \mathrm{~km})$ made with the Poker Flat Incoherent Scatter Radar (PFISR). Using long uncoded pulses, their range resolution was limited to $75 \mathrm{~km}$, which roughly coincided with the altitude range of the enhancements. It is therefore likely that the process generating the enhancements was more localised than what was resolved. Power enhancements of 3-5 times the thermal level were seen and the enhanced spectra showed substantial power enhancement also between the ion line shoulders. Both the associated power enhancements and the altitude extent differ from many NEIAL observations as described above. A recent PFISR study (Michell and Samara, 2010) identified four cases of ion line enhancements between $150 \mathrm{~km}$ and $400 \mathrm{~km}$ altitude correlated with auroral arcs. None of these cases showed significant asymmetry between the upand downshifted shoulders, and the spectral region between them was also enhanced. This type of ion line enhancement is possibly similar to those observed by Rietveld et al. (2002) with the EISCAT UHF during a heating campaign. Rietveld et al. recorded an enhanced ion line, which did not correlate with the HF modulation and originated from a thin region that was descending from $310 \mathrm{~km}$ to $280 \mathrm{~km}$ altitude. The observation was interpreted as a narrow region of natural plasma instability, possibly related to an auroral 
arc intersecting the radar beam.

The broad-band character of these observations cannot be easily explained in terms of current NEIAL models. Michell et al. (2008) suggested a possible connection to broad-band extremely low-frequency (BBELF) wave activity, based on the auroral morphology accompanying their observations. However, the enhancements reported by Michell et al. $(2008,2009)$ and Michell and Samara (2010), where both ion-acoustic shoulders and the spectral region between them are enhanced equally and simultaneously, can also be replicated by the recently published spectral-shape-conserving soliton-induced ion line enhancement model by Ekeberg et al. (2010).

Here we analyse observations from the ESR, taken during the International Polar Year (IPY) between March 2007 and February 2008. The experiment used a coded modulation, which enables a study of spectra with high spatial and spectral resolutions. We present 48 ion line enhancement events recorded with $4.5 \mathrm{~km}$ altitude resolution, together with the corresponding background electron density and temperature profiles. The events are shown to have an increased likelihood of occurrence during magnetically disturbed conditions and they nearly always occur at the ionospheric $F$ region peak, which is a prerequisite for the applicability of the model by Ekeberg et al. (2010). We describe the characteristics of the enhanced spectra, study the $K$ index and magnetic local time dependence and relate the altitude of their occurrence to the $F$ peak.

\section{Observation}

The ESR (Wannberg et al., 1997) is a $500 \mathrm{MHz}$ incoherent scatter radar located on Spitsbergen at $78^{\circ} 09^{\prime} 11^{\prime \prime} \mathrm{N}$, $16^{\circ} 01^{\prime} 44^{\prime \prime} \mathrm{E}$. The system comprises two parabolic dish antennae of $32 \mathrm{~m}$ and $42 \mathrm{~m}$ in diameter. The 32-m dish is fully steerable in azimuth and elevation, whereas the 42-m dish is fixed along the local direction of the geomagnetic field with an azimuth of $181^{\circ}$ and an elevation of $81.6^{\circ}$.

Beginning in March 2007, the IPY experiment was carried out using both dishes of the ESR. The experiment used a $32 \times 30 \mu$ s alternating code (Lehtinen and Häggström, 1987 ) with maximum range and time resolutions of $4.5 \mathrm{~km}$ and $6 \mathrm{sec}$, respectively. It was run until the end of February 2008 and focused on lower heights $(<500 \mathrm{~km})$.

\section{Analysis}

All 5450 hours of the ESR IPY data from the 42-m dish have been analysed according to the method described below. First, the raw data from the $200-400 \mathrm{~km}$ altitude interval were integrated to a spatial and temporal resolution of $18 \mathrm{~km}$ and $6 \mathrm{sec}$, respectively. This was done using the EISCAT standard analysis software package GUISDAP (Lehtinen and Huuskonen, 1996). In order to exclude noisy spectra and satellite echoes, a noise baseline proxy was defined by integrating the total power well outside the ion line, i.e. in the spectral windows $[-33,-10] \mathrm{kHz}$ and $[10,33]$ $\mathrm{kHz}$.

The maximum power spectral density $\mathrm{PSD}_{\max }$ and the ratio between the power in the ion line window $[-7,7] \mathrm{kHz}$ and the baseline proxy, PR, were then computed for each height. In order to identify the most pronounced enhance- ments, only those spectra where at least one range gate exhibited $\mathrm{PR}>6$ and $\mathrm{PSD}_{\max }>2 \mathrm{mK} / \mathrm{Hz}$ were selected. With a system noise temperature of $70 \mathrm{~K}$ over the $67 \mathrm{kHz}$ bandwidth, $\mathrm{PSD}_{\max }=2 \mathrm{mK} / \mathrm{Hz}$ corresponds to a signal-tonoise ratio of $200 \%$. This sub-group was then re-integrated with the highest available range resolution, $4.5 \mathrm{~km}$, and only those spectra where a single range gate still exhibited $\mathrm{PR}>6$ were retained. Two-minute time windows centred on each of these enhancements were then searched for additional enhancements; if a window was found to contain at least two more 6-second intervals with PR $>6$ and $\mathrm{PSD}_{\max }>0.4 \mathrm{mK} / \mathrm{Hz}$, the event was retained. Using these criteria, we identified 83 enhanced spectra.

Spectra within 1 minute of one another were considered to be part of the same event. All but the first pronounced enhancement among such multiplets were removed. For each of the remaining spectra, average profiles of electron density, electron temperature and ion temperature were integrated for the $10 \mathrm{~min}$ preceding the pronounced enhancement. The difference in height between the spectral enhancement and the local maximum at the ionospheric $F$ region peak was calculated. 48 events of spectral enhancements and their corresponding average profiles of plasma parameters remained. As suggested by the observations of Michell et al. (2008, 2009) and Michell and Samara (2010), there is a possible connection between auroral activity and the type of ion line enhancements presented here. The $K$ index is a measure of magnetic disturbance and in order to quantify a possible relation to auroral activity, the $K$ index was noted for each event.

A spectrally uniform ion line enhancement will conserve the ion line shape, i.e. it will leave the relative levels of different spectral bins in the ion line unchanged. The degree of enhancement uniformity was examined by comparing the relative changes of the power spectral densities at five frequencies evenly distributed between the ion line shoulders to the relative change in total spectral power contained in the ion line over time. Choosing a significance level of 5\%, the correlation coefficient between the two sets of ratios was calculated and a least squares linear regression was carried out.

\section{Results}

The right panel of Fig. 1 shows spectra in one of the events identified in Section 3 observed on 5 April 2007 at 17:14:30 UT; the left panel displays the corresponding pre-event average profiles of electron density, ion and electron temperature and electron-to-ion temperature ratio. The spectral enhancement is localised in height to an altitude range of less than $15 \mathrm{~km}$, occurs close to the ionospheric $F$ region peak and is relatively uniform over the spectral window of the ion line. All these characteristics are different from classical NEIAL observations. The observational differences between a classical NEIAL and the present type of enhancement are exemplified by Fig. 1 in Ekeberg et al. (2010). The ion and electron temperatures of respectively $910 \mathrm{~K}$ and $2100 \mathrm{~K}$ seen at the density peak both represent typical $F$ region conditions. The electron density at the $F$ region peak was $2.4 \times 10^{11} \mathrm{~m}^{-3}$.

Figure 2 shows the time evolution of the spectra mea- 

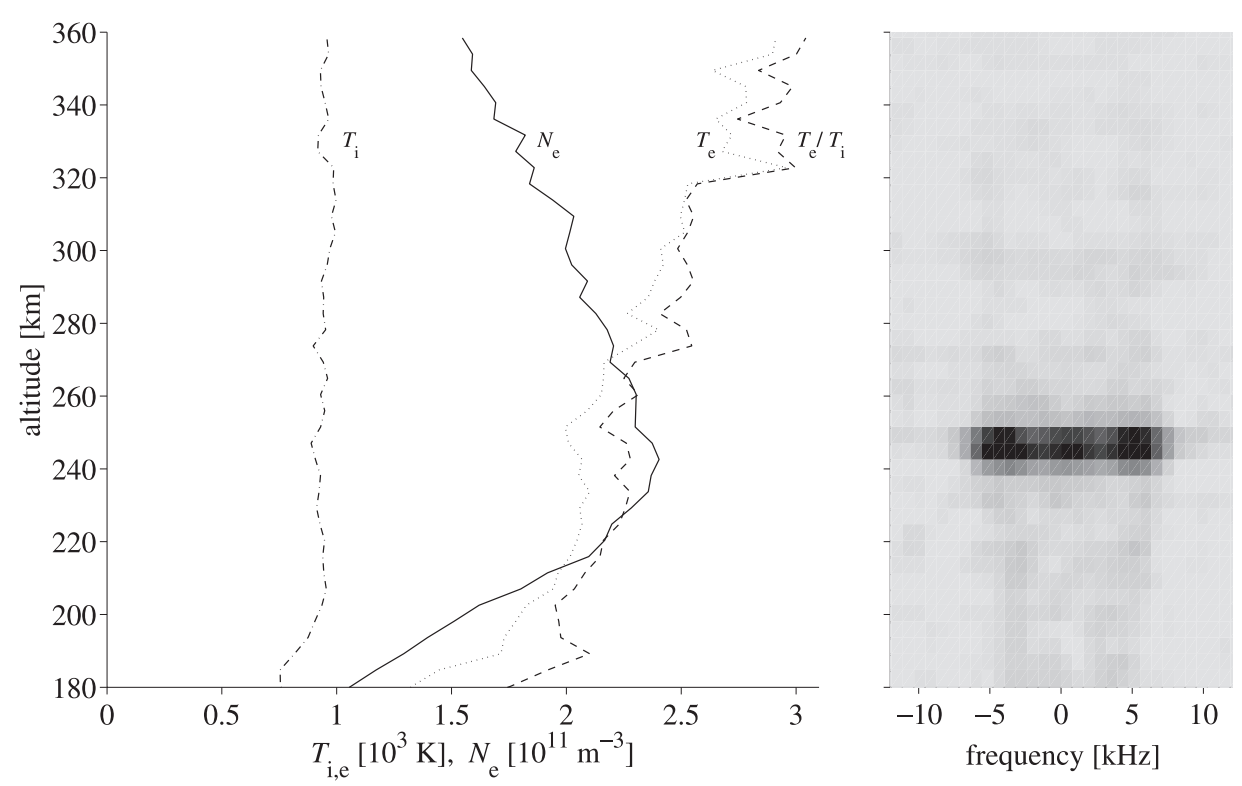

Fig. 1. ESR data from 5 April 2007. (Left) Profiles of ion and electron temperature $T_{\mathrm{i}, \mathrm{e}}$, electron density $N_{\mathrm{e}}$ and electron to ion temperature ratio averaged over 17:04:30-17:14:30 UT. (Right) 6-second average range corrected power spectral density at 17:14:30 UT coded with a grey scale.
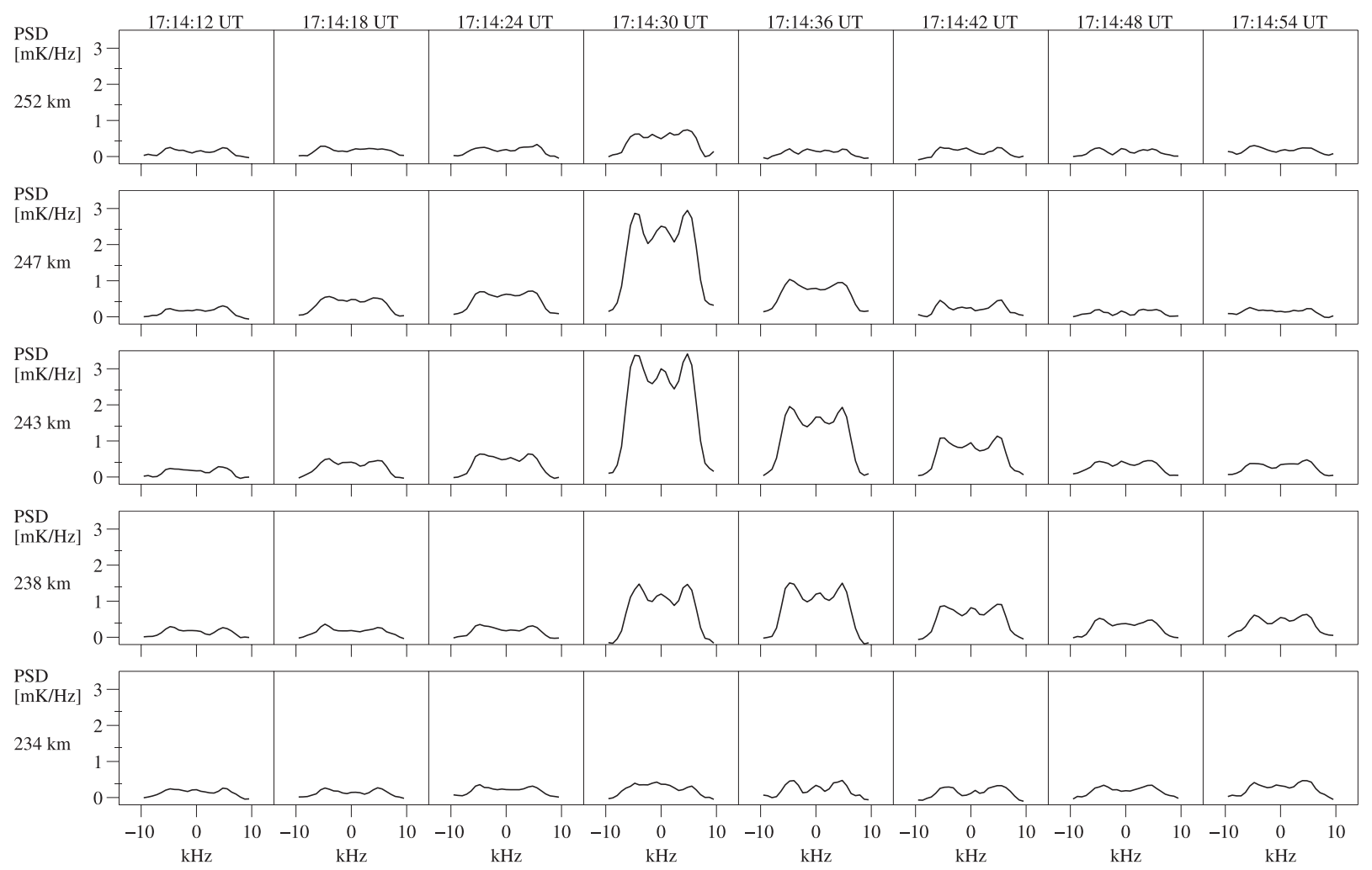

Fig. 2. Incoherent scatter radar spectra measured with the $42 \mathrm{~m}$ ESR on 5 April 2007. The full time resolution of 6 sec is used and the starting times for each integration are shown for 5 adjacent height gates, which are centred at the altitudes indicated. The $x$ axes show frequency and the $y$ axes show range corrected power spectral density. The spectra at 17:14:30 UT over a longer height range are shown in Fig. 1.

sured on 5 April 2007 between 17:14:12 UT and 17:15:00 UT in the altitude range $234-252 \mathrm{~km}$. At 17:14:30 UT (also shown in Fig. 1), the integrated scattering cross sections at 238, 243 and $247 \mathrm{~km}$ are enhanced by 6,15 and 13 times relative to the background level (17:14:12 UT), respectively. The spectral enhancement is relatively uniform but displays a small central peak. Almost no enhancement is seen outside the three middle height gates, corresponding to an altitude range of $14 \mathrm{~km}$. The duration of the major enhancement is less than 30 seconds.

A comparison of the relative changes in the power spectral densities $(y)$ to the relative changes in the spectral powers $(x)$ contained in the observed ion lines was carried out according to the procedure described in Section 3. This gave a correlation coefficient of 0.97 , the regression equation $y=(1.2 \pm 0.064) x-0.19 \pm 0.33$ with indicated $95 \%$ 
Table 1. Statistics on the 48 spectral enhancement events showing event duration, enhancement above the thermal level, altitude of occurrence and deviation from the $F$ region peak (positive above the peak). The four plasma parameters, based on 10 min averaged profiles preceding the events, are given at the $F$ peak. * Value has been rounded from 1.4.

\begin{tabular}{lccccc}
\hline & Max & Min & Mean & Median & Standard dev. \\
\hline Duration [sec] & 230 & 20 & 80 & 60 & 50 \\
Enhancement & 40 & $1^{*}$ & 7 & 6 & 7 \\
Altitude $[\mathrm{km}]$ & 280 & 210 & 230 & 230 & 20 \\
Deviation $[\mathrm{km}]$ & 50 & -40 & 0.5 & 0.1 & 10 \\
$N_{\mathrm{e}}\left[10^{11} \mathrm{~m}^{-3}\right]$ & 4.5 & 1.2 & 2.2 & 2.3 & 0.64 \\
$T_{\mathrm{e}}\left[10^{3} \mathrm{~K}\right]$ & 2.8 & 1.0 & 2.2 & 2.2 & 0.30 \\
$T_{\mathrm{i}}\left[10^{3} \mathrm{~K}\right]$ & 1.4 & 0.80 & 0.99 & 0.96 & 0.13 \\
$T_{\mathrm{e}} / T_{\mathrm{i}}$ & 2.8 & 1.2 & 2.3 & 2.3 & 0.40 \\
\hline
\end{tabular}

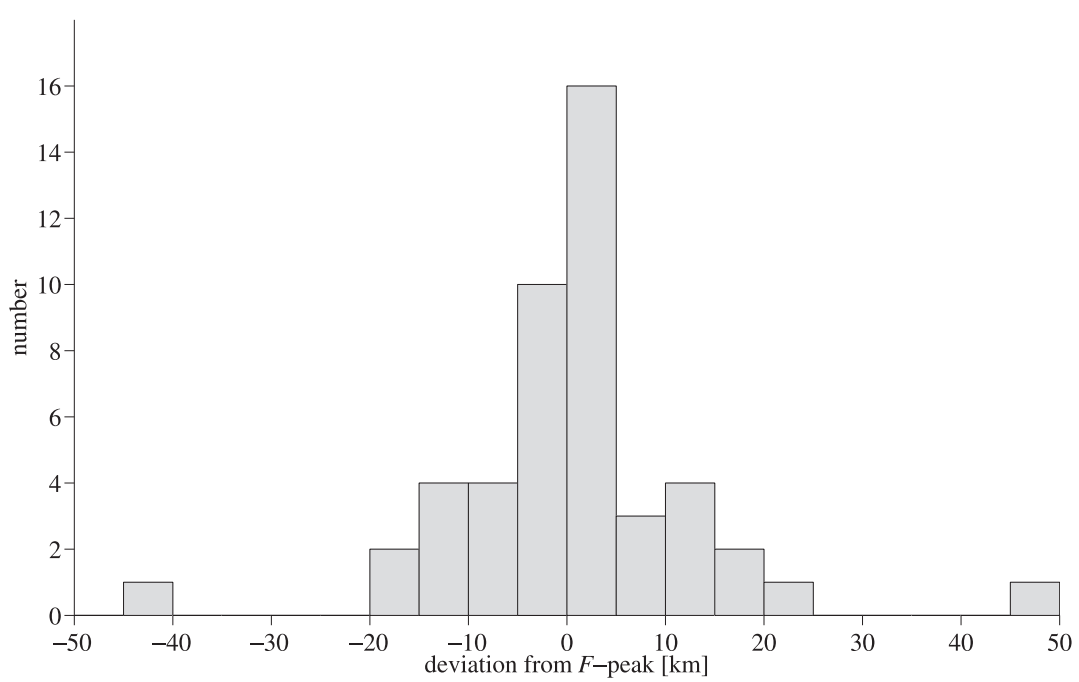

Fig. 3. Distribution of altitude deviations from the $F$ peak for the first spectra with a power ratio PR $>6$ in the 48 events. The $y$ axis shows the number of events for $205-\mathrm{km}$-sized bins. Positive and negative deviations represent spectral enhancements occurring respectively above and below the peak.

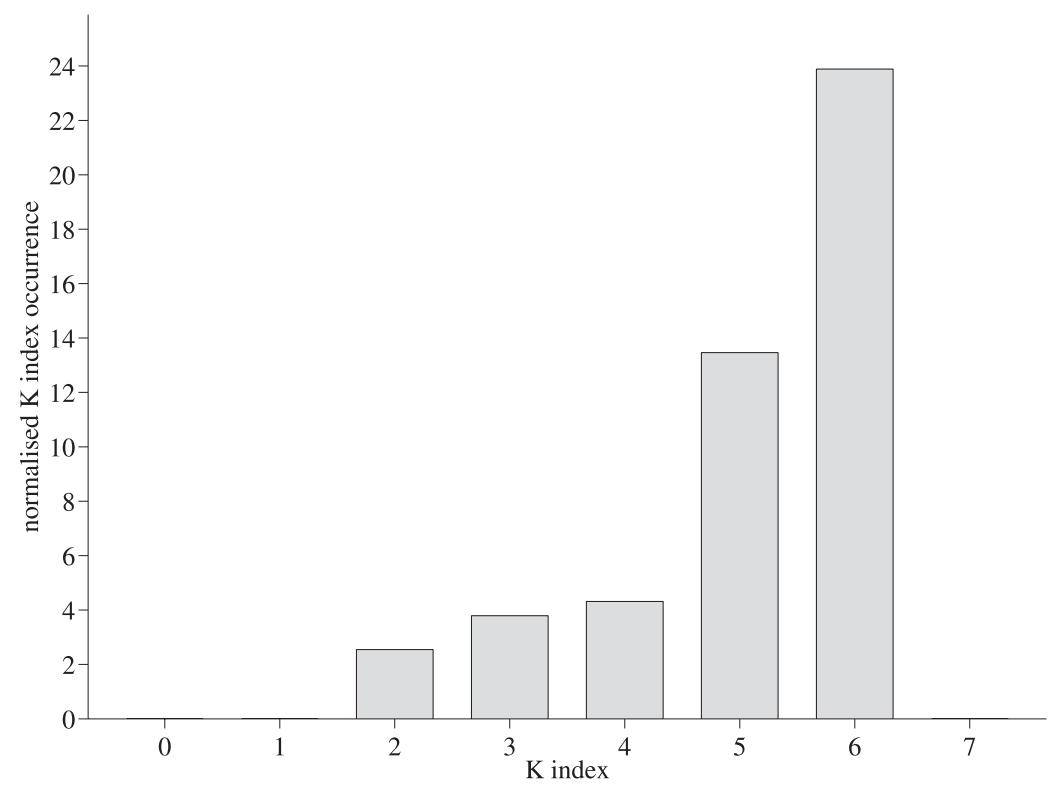

Fig. 4. Normalised $K$ index occurrence for the 48 events.

confidence limits, and an associated coefficient of determination of $r^{2}=0.95$.

A statistical overview of the 48 events is shown in Table 1. The duration of an event was defined as the longest time interval, where spectra with PR $>6$ were separated by less than 1 minute. The spectral enhancement above the thermal level, occurrence altitude and vertical deviation from the ionospheric $F$ region peak (positive above the 


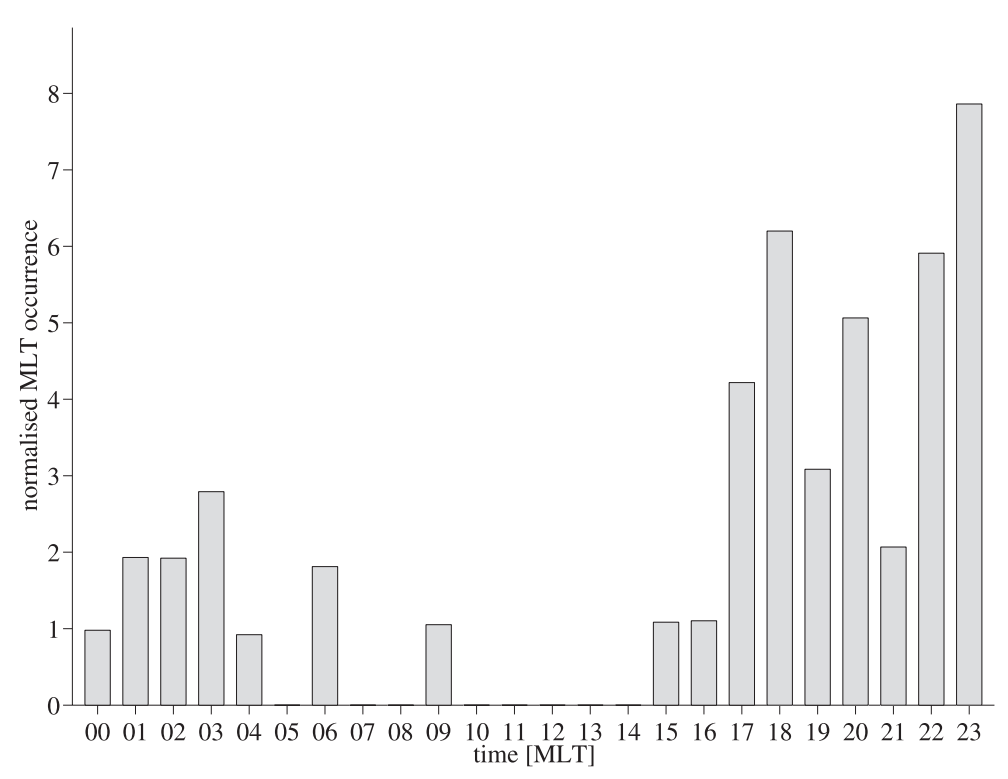

Fig. 5. Normalised MLT occurrence for the 48 events.

peak) are given for the first spectra in each event having $\mathrm{PR}>6$. $F$ region peak values of electron density, electron and ion temperatures and the electron-to-ion temperature ratio are based on $10 \mathrm{~min}$ average profiles preceding the events.

Figure 3 shows the altitude separation between the ionospheric $F$ region peak and the height at which the first enhanced $(P R>6)$ spectrum in an event was seen. The first enhanced spectrum is used rather than the one with maximum enhancement in order to eliminate as far as possible any bias resulting from the process causing the enhancement. Positive and negative deviations represent events occurring respectively above and below the $F$ region peak. As can be seen, more than $50 \%$ of the events occur within $5 \mathrm{~km}$ from the peak and $85 \%$ occur within 1 standard deviation.

Figure 4 shows the occurrence of SUIPEs as a function of $K$ index. The occurrence has been normalised to the total occurrence of the respective $K$ values over the year in the analysed data. It is clear that SUIPEs are more likely to occur during magnetically disturbed times.

Figure 5 presents the event occurrence as a function of hour in magnetic local time (MLT). The occurrence rate has been normalised to the amount of analysed data for each respective hour. It can be seen that SUIPEs preferentially occur during magnetic local evening to midnight with $72 \%$ of the events taking place between 17 MLT and 00 MLT. During the subsequent 7-h interval (00-07 MLT) 22\% of the events are found. Between 10 MLT and 15 MLT, no events were seen.

\section{Discussion}

We have identified and studied 48 spectrally uniform ion line power enhancements (SUIPEs) distributed over a contiguous 12-month period. As seen in Table 1, the SUIPEs are observed at altitudes between 210 and $280 \mathrm{~km}$ with a mean and a standard deviation of $230 \mathrm{~km}$ and $20 \mathrm{~km}$, respectively. The enhancements are one order of magnitude above the thermal level. The high correlation coefficient
(0.97) between the relative changes in the power spectral densities of the ion line $(y)$ and the ion line integrated power $(x)$ and the regression analysis show a close resemblance of a one-to-one relation between $y$ and $x$. This indicates a high degree of spectral enhancement uniformity, in other words, the generation mechanism enhances both shoulders and the spectral region between them simultaneously and equally. Event durations of the order of minutes rule out the possibility of the signatures being associated with satellites. The mean values of electron density and electron and ion temperatures at the $F$ region peak preceding the events are respectively $2.2 \times 10^{11} \mathrm{~m}^{3}, 2200 \mathrm{~K}$ and $990 \mathrm{~K}$, representing typical conditions in the $F$ region.

An example sequence of spectra taken on 5 April 2007 between 17:14:18 UT and 17:14:54 UT showed a scattering cross section enhancement at $243 \mathrm{~km}$ altitude of more than 7 times the value at the beginning of the time interval. The corresponding cross section enhancement in the neighbouring height gates $(238$ and $247 \mathrm{~km}$ ) reached a maximum of 5 times.

As shown in Fig. 3, the enhanced spectra are essentially co-located with the ionospheric $F$ region peak. The average deviation is $0.5 \mathrm{~km}$ and more than $50 \%$ of the cases occur within $5 \mathrm{~km}$ of the peak and $85 \%$ within 1 standard deviation $(10 \mathrm{~km})$. The spectral enhancements are localised in height and are often concentrated to an altitude range of less than $20 \mathrm{~km}$. None of the current NEIAL models have been shown to generate a broad-band spectral enhancement and have not been related to the $F$ region peak. However, the fact that the events occur at the $F$ region peak supports a recently published model (Ekeberg et al., 2010), where solitary structures generated by magnetic field perturbations were suggested as a possible generation mechanism for the type of uniform enhancements presented here. Supported by a pumping electron beam, the one-dimensional model by Ekeberg et al. (2010) describes how compressive solitary structures could transport plasma into the radar beam and enhance the scattering cross section. In the absence of ver- 
tical gradients, the model is valid over a range of altitudes. This condition is fulfilled in the vicinity of the $F$ region peak, which could explain the strong localisation of the observed enhancements. The model parameters are representative of those seen in the present observations and predict enhancements of one order of magnitude above the thermal level, in good agreement with the observations.

Figure 4 shows that SUIPEs are more likely to occur during magnetically disturbed times, possibly related to auroral activity in the vicinity of the radar beam. This is in agreement with the observations of Rietveld et al. (2002) and Michell and Samara (2010), where the spectral enhancements were seen either during generally enhanced auroral activity or were directly correlated with auroral arcs intersecting the radar beam. Since electron beams and a perturbed magnetic field are both features of active aurora and also prerequisites for the Ekeberg et al. (2010) model mentioned above, auroral activity may drive solitary structure formation and subsequent SUIPE generation.

In Fig. 5, it is seen that SUIPEs preferentially occur during magnetic local evening to midnight with $72 \%$ of the events taking place between 17 MLT and 00 MLT. The spectral enhancements presented by Michell et al. (2008, 2009) and Michell and Samara (2010) are similar to those in this study and all occurred between 20 MLT and 00 MLT. It should be stated, however, that in those studies, only magnetic nightside radar data were considered.

The spectral enhancements reported by Michell et al. (2008) and Michell and Samara (2010) were not accompanied by pronounced $F$ region density peaks. Instead, the authors related the spectral enhancements to the background electron densities. Their reported electron densities ( 5 to $30 \times 10^{10} \mathrm{~m}^{-3}$ ) match the lower end of the range of $F$ peak densities given in Table 1. Although, there may be a threshold electron density for generating this type of spectral enhancement, this is not sufficient to explain the strong co-location with the $F$ peak, as exemplified by Fig. 1. However, the spectra in the studies of Michell et al. (2008) and Michell and Samara (2010) were averaged over a $70 \mathrm{~km}$ altitude range and the enhancements were centred on altitudes likely to bracket the $F$ region peak. We therefore suggest that their observed spectral enhancements are similar to those presented here and possibly generated by the Ekeberg et al. (2010) soliton mechanism.

Many of the enhanced spectra exhibit a small central peak at or close to zero Doppler shift, i.e. symmetrically located between the ion line shoulders. Such peaks are frequently seen in ionospheric HF heating experiments (Stubbe et al., 1992) and in some NEIAL observations (Forme et al., 1995). The central peak has been studied in numerical models of Langmuir turbulence, where it has been interpreted as due to scattering off stationary density structures (e.g., DuBois et al., 1991; Guio and Forme, 2006) or due to the modulational instability (Sprague and Fejer, 1995). However, whereas signatures of the modulational instability are always present and strong in EISCAT UHF (931 MHz) observations, they are weak or undetectable in EISCAT VHF (224 MHz) observations. The present study is based on data from the ESR which operates at $500 \mathrm{MHz}$. It is not clear how pronounced the effects of a modulational instability are expected to be at this intermediate spatial scale. Nevertheless, in a region with weak density gradients, the diffusion time, and therefore the lifetime of density structures, is long. Therefore, the near absence of vertical density gradients near the $F$ region peak will support both transversely propagating solitary structures and stationary structures. As can be seen in the time sequence in Fig. 2, there are, even though sometimes very weak, signs of a central peak in many of the spectra, both before and after the most pronounced enhancement. We interpret this as that the central peak was present before the enhancement process was initiated. The enhancement mechanism suggested by Ekeberg et al. (2010) would equally and simultaneously enhance both ion-acoustic shoulders and the spectral region between them, including the central peak, and therefore conserve the spectral shape.

\section{Conclusions}

We present an analysis of 48 spectrally uniform ion line power enhancement events, extracted from 5450 hours of ESR IPY incoherent-scatter data using stringent selection criteria. The enhancements are statistically demonstrated to be uniform, in that both ion-acoustic shoulders as well as the spectral region between them are enhanced simultaneously and equally by one order of magnitude above the thermal level. Most events occur between local magnetic evening and local magnetic midnight. There is a clear preference for this type of uniform power enhancement to occur during magnetically disturbed times, possibly indicating an association with auroral activity in the vicinity of the radar beam.

Most importantly, the enhancement events are also statistically demonstrated to be essentially co-located with the ionospheric $F$ region peak, with a standard deviation of only $10 \mathrm{~km}$ or about twice the altitude resolution of the IPY experiment. This fact strongly supports the solitary wave enhancement mechanism hypothesis first suggested and modelled by Ekeberg et al. (2010). The virtual absence of vertical electron density gradients in the immediate vicinity of the $F$ peak makes this altitude region optimal for the formation of vertically extended compressive solitary waves, which propagate transversely across the geomagnetic field and carry compressed plasma into the radar beam, thereby enhancing the whole ion line uniformly by one order of magnitude above the thermal level.

Acknowledgments. EISCAT is an International Association supported by Finland (SA), France (CNRS), Germany (MPG), Japan (NIPR), Norway (NFR), Sweden (VR) and the United Kingdom (PPARC). J. Ekeberg is grateful to Dr. M. Vogt for valuable input.

\section{References}

Buchert, S. C., A. P. van Eyken, T. Ogawa, and S. Watanabe, Naturally enhanced ion-acoustic lines seen with the EISCAT Svalbard radar, $A d v$. Space Res., 23, 1699-1704, 1999.

Collis, P. N., I. Häggström, K. Kaila, and M. T. Rietveld, EISCAT radar observations of enhanced incoherent scatter spectra: Their relation to red aurora and field-aligned currents, Geophys. Res. Lett., 18, 10311034, 1991.

DuBois, D. F., H. A. Rose, and D. Russell, Coexistence of parametric decay cascades and caviton collapse at subcritical densities, Phys. Rev. Lett., 66, 1970-1973, 1991.

Ekeberg, J., G. Wannberg, L. Eliasson, and K. Stasiewicz, Ion-acoustic 
solitary waves and spectrally uniform scattering cross section enhancements, Ann. Geophys., 28, 1299-1306, 2010.

Forme, F. R. E., D. Fontaine, and J. E. Wahlund, Two different types of enhanced ion acoustic fluctuations observed in the upper ionosphere, J. Geophys. Res., 100, 14625-14636, 1995.

Foster, J. C., C. del Pozo, K. Groves, and J. P. St-Maurice, Radar observations of the onset of current-driven instabilities in the topside ionosphere, Geophys. Res. Lett., 15, 160-163, 1988.

Grydeland, T., E. Blixt, U. Løvhaug, T. Hagfors, C. La Hoz, and T. Trondsen, Interferometric radar observations of filamented structures due to plasma instabilities and their relation to dynamic auroral rays, Ann. Geophys., 22, 1115-1132, 2004.

Guio, P. and F. Forme, Zakharov simulations of Langmuir turbulence: Effects on the ion-acoustic waves in incoherent scattering, Phys. Plasmas, 13, 122,902, 2006.

Lehtinen, M. S. and I. Häggström, A new modulation principle for incoherent scatter measurements, Radio Sci., 22, 625-634, 1987.

Lehtinen, M. S. and A. Huuskonen, General incoherent scatter analysis and GUISDAP, J. Atmos. Terr. Phys., 58, 435-452, 1996.

Michell, R. G. and M. Samara, High-resolution observations of naturally enhanced ion acoustic lines and accompanying auroral fine structures, J. Geophys. Res., 115, 3310, 2010.

Michell, R. G., K. A. Lynch, C. J. Heinselman, and H. C. StenbaekNielsen, PFISR nightside observations of naturally enhanced ion acoustic lines, and their relation to boundary auroral features, Ann. Geophys., 26, 3623-3639, 2008.

Michell, R. G., K. A. Lynch, C. J. Heinselman, and H. C. StenbaekNielsen, High time resolution PFISR and optical observations of naturally enhanced ion acoustic lines, Ann. Geophys., 27, 1457-1467, 2009.

Ogawa, Y., S. C. Buchert, R. Fujii, S. Nozawa, and F. Forme, Naturally enhanced ion-acoustic lines at high altitudes, Ann. Geophys., 24, 33513364, 2006.

Rietveld, M. T., P. N. Collis, and J. P. St-Maurice, Naturally enhanced ionacoustic waves in the auroral ionosphere observed with the EISCAT 933 MHz radar, J. Geophys. Res., 96, 19291-19305, 1991.

Rietveld, M. T., P. N. Collis, A. P. van Eyken, and U. P. Løvhaug, Coherent echoes during EISCAT UHF Common Programmes, J. Atmos. Terr. Phys., 58, 161-174, 1996.

Rietveld, M. T., B. Isham, T. Grydeland, C. La Hoz, T. B. Leyser, F. Honary, H. Ueda, M. Kosch, and T. Hagfors, HF-Pump-induced parametric instabilities in the auroral E-region, Adv. Space Res., 29, 13631368, 2002.

Rosenbluth, M. N. and N. Rostoker, Scattering of electromagnetic waves by a nonequilibrium plasma, Phys. Fluids, 5, 776-788, 1962.

Sedgemore-Schulthess, F. and J. St.-Maurice, Naturally Enhanced IonAcoustic Spectra and Their Interpretation, Surv. Geophys., 22, 55-92, 2001.

Sprague, R. A. and J. A. Fejer, Simultaneous excitation of parametric decay cascades and of the oscillating two-stream instability in onedimensional numerical simulations based on Zakharov's equations, J. Geophys. Res., 100, 23959-23972, 1995.

Stubbe, P., H. Kohl, and M. T. Rietveld, Langmuir turbulence and ionospheric modification, J. Geophys. Res., 97, 6285-6297, 1992.

Wannberg, G. et al., The EISCAT Svalbard radar: A case study in modern incoherent scatter radar system design, Radio Sci., 32, 2283-2308, 1997.

J. Ekeberg (e-mail: jonas.ekeberg@irf.se), G. Wannberg, L. Eliasson, and I. Häggström 